\title{
Patterns of Integrative Korean Medicine Practice for Anxiety Disorders: A Survey among Korean Medicine Doctors (KMDs) in Korea
}

\author{
Sang-Il Seo, ${ }^{1}$ Sung-Youl Choi, ${ }^{2}$ Eun-Jung Kim $\left(\mathbb{D},{ }^{3}\right.$ Byung-Soo Koo, ${ }^{4}$ Hyo Won-Jung, ${ }^{5}$ \\ Gun-Hee Lee, ${ }^{6}$ and Geun-Woo Kim $\mathbb{D}^{4}$ \\ ${ }^{1}$ Department of Neuropsychiatry, Dong-Guk University Bundang Korean Medicine Hospital, 268, Buljeong-ro, Bundang-gu, \\ Seongnam-si, Gyeonggi-do 13601, Republic of Korea \\ ${ }^{2}$ Department of Neuropsychiatry, College of Korean Medicine, Gachon University, 1342, Seongnam-daero, Sujeong-gu, \\ Seongnam-si, Gyeonggi-do 13120, Republic of Korea \\ ${ }^{3}$ Department of Acupuncture \&Moxibustion, College of Korean Medicine, Dong-Guk University, 32, Dongguk-ro, Ilsandong-gu, \\ Goyang-si, Gyeonggi-do 10326, Republic of Korea \\ ${ }^{4}$ Department of Neuropsychiatry, College of Korean Medicine, Dong-Guk University, 32, Dongguk-ro, Ilsandong-gu, Goyang-si, \\ Gyeonggi-do 10326, Republic of Korea \\ ${ }^{5}$ Department of Herbology, College of Korean Medicine, Dong-Guk University, 123, Dongdae-ro, Gyeongju-si, \\ Gyeongsangbuk-do 38066, Republic of Korea \\ ${ }^{6}$ Sogang Business School, Sogang University, 35, Baekbeom-ro, Mapo-gu, Seoul 04107, Republic of Korea
}

Correspondence should be addressed to Geun-Woo Kim; kgwoo86@hanmail.net

Received 15 March 2020; Revised 23 July 2020; Accepted 5 August 2020; Published 5 October 2020

Academic Editor: Morry Silberstein

Copyright ( 2020 Sang-Il Seo et al. This is an open access article distributed under the Creative Commons Attribution License, which permits unrestricted use, distribution, and reproduction in any medium, provided the original work is properly cited.

Anxiety disorder is known as the most common disease among psychiatric disorders. However, many studies have not been conducted in the Korean medicine area. This study explores the current state of anxiety disorder treatments of Korean medicine through a survey research. The survey for Korean medicine doctors (KMDs) on Korean medicine (KM) diagnosis and treatments for anxiety disorder was conducted online from December 21, 2016, to December 29, 2016. The results were divided into two groups, KMDs and Korean medicine neuropsychiatric specialists (KMNPS), and comparatively analyzed. Self-evaluation and counseling were the most common in both diagnostic methods and evaluation of treatment effects, and KMNPS tended to make extensive use of objective indicators. There was no difference in the rate of psychiatric medication use among the patients between KMD and KMNPS. The main reason for patients wanting KM treatment was the tapering cessation of psychiatric medications. The most common treatments were acupuncture, herbal medicine, and moxibustion, in addition to dry cupping in KMD and psychotherapy in KMNPS. The most important factor for treatment was herbal medicine treatment, followed by rapport formation in KMD and patient's temperament in KMNPS. Opinions on various items were presented as treatment barriers, and KMNPS tended to think more importantly about the patient's family problems. For the items to be additionally trained in the future, KMD chose the diagnostic tools and KMNPS chose psychotherapies. This study is the first study to analyze the clinical patterns for anxiety disorder in KMDs. KMD and KMNPS showed similar patterns in the perception, diagnosis, and treatment of anxiety disorders, but KMNPS tended to use objective indicators and psychotherapy more actively. Further clinical studies for the development of clinical guidelines should be additionally required. 


\section{Introduction}

Anxiety disorder is the most common disease among psychiatric disorders. According to the WHO report, the proportion of the global population with anxiety disorders in 2015 is estimated to be $3.6 \%$ [1], and the total estimated number of people living with anxiety disorders in the world is 264 million. This total for 2015 reflects a $14.9 \%$ increase since 2005 [2]. A systematic analysis showed that the pooled period prevalence was $6.7 \%(6.0-7.6 \%)$ from 122 surveys with a lifetime prevalence of $12.9 \%(11.3-14.7 \%)$ from 70 surveys [3]. It is also shown that the lifetime prevalence of anxiety disorders in Korea has been reported as 9.3\% (male $6.7 \%$ and female $11.7 \%$ ) with the annual prevalence rate at $5.7 \%$ (male $3.5 \%$ and female $7.5 \%$ ). That is almost two times higher than depression, at lifetime prevalence of 5.4\% (male $3.3 \%$ and female $7.2 \%$ ), with an annual prevalence rate of $3.1 \%$ (male $1.8 \%$ and female $4.3 \%$ ) according to the Epidemiological Survey of Mental Disorders. In addition, the lifetime prevalence of all mental illnesses decreased from $29.9 \%$ in 2001 to $26.6 \%$ in 2016 , but the lifetime prevalence of "anxiety disorder" has increased to $6.9 \%$ in $2006,8.7 \%$ in 2011 , and $9.3 \%$ in 2016 [4]. However, the lifetime use rate of mental health services for patients with anxiety disorders is still low at $25.1 \%$ in 2011 and $27.3 \%$ in 2016 [5].

Korean medicine doctor (KMD) is a Korean medical practitioner who provides diagnosis, treatment, and prescription and necessary medical consultations to maintain the desired level of health [6]. A Korean medicine specialist is a person who is certificated in oriental medicine and has acquired professional qualifications through one year of internship with three years of residence training after 2000. Currently, specialists are trained in 8 subject areas (Internal Korean Medicine, Acupuncture and Moxibustion Medicine, KM Obstetrics and Gynecology, KM Pediatrics, KM Rehabilitation, KM Neuropsychiatry, Sasang Constitutional Medicine, KM Ophthalmology, Otolaryngology and Dermatology). KM specialists accounted for about $9.1 \%$ of all KM doctors by 2013 [7]. Training subjects of Korean medicine neuropsychiatry specialist (KMNPS) include psychophysiology, psychopathology, psychopharmacology, neurology with several other psychological-psychiatric theories and techniques, psychiatric examination, differential diagnosis, psychological testing, specialized KM therapies for neuropsychiatric disorders, and KM psychotherapies such as Qi-gong and meditation, Korean traditional psychological-psychiatric theories, personality theories, and techniques [8].

There are many types of psychiatric illnesses situated in KM institutions that are mostly related to physical symptoms. KM, which has a symptom-based approach to determine the treatment through the identification of specific etiology or specific symptoms rather than prioritizing the name of the disease [9], may have an advantage in anxiety disorders, which indicate the diversity of symptoms among psychiatric disorders. In fact, complementary and alternative medicine treatments including KM and Chinese medicine are used worldwide [10]. However, the standard of care has not been established due to subjective diagnosis and various treatment methods. Therefore, the pursuit of evidence-based care is a very important topic, and the demand for clinical practice guidelines (CPG) is increasing. In the Chinese version of the Evidence-Based Guidelines for Clinical Practice in Chinese Medicine, depressive disorders and insomnia CPG are mentioned, but none exists for anxiety disorders [11].

The purpose of this study is to analyze the KM treatment pattern of anxiety disorders and reflect the data in developing KM CPG for anxiety disorders. Also, the study is to investigate the possibility of clinical application of $\mathrm{KM}$ therapy tools based on the Diagnosis and Statistical Manual for Mental Disorders (DSM-IV TR and DSM-5) and International Classification of Diseases (ICD-10), after that the questionnaire consists of panic disorder (PD), posttraumatic stress disorder (PTSD), social anxiety disorder (SAD), and generalized anxiety disorder (GAD). Finally, we expect that this study will contribute to the standardization and efficiency improvement of KM treatment for anxiety disorders, enabling KMDs to provide the best medical care.

\section{Materials and Methods}

2.1. Study Design. This questionnaire was prepared for KMD and KMNPS to understand the perception and treatment of anxiety disorders. SIS searched for literature published within the last 10 years with the search formula ["anxiety disorder" or "generalized anxiety disorder" or "panic disorder" or "post-traumatic stress disorder" or "social phobia"] and ["professional" or "specialist" or "therap"”] and "survey" in PubMed. Of the 219 search results, 12 relevant studies were selected and referenced in the production of the questionnaire [12-23]. Three KMNPS (SIS, SYC, and GWK) and one acupuncture and moxibustion medicine specialist (EJK), totaled four KMDs, participated in drafting. The questionnaire covers demographic characteristics, diagnosis method, treatment method, assessment method, and factors affecting the treatment of anxiety disorders. The draft was made after two conferences. One KMNPS (BSK) and one KMD (HWJ), who had not participated in the drafting, participated in revision. The revised version was finalized after consulting with KMNPS and statisticians who did not participate in the questionnaire production. The Supplementary Material (available here) shows the entire survey. This study was approved by the Institutional Review Board of Dongguk University Bundang Korean Hospital in Korea (DUBHIRB2017-0012).

2.2. Participants and Procedures. The questionnaire was distributed to $18,329 \mathrm{KMDs}$ and $165 \mathrm{KMNPS}$ registered through the Association of Korean Medicine. We used "Surveymonkey," an online survey platform, and the survey was conducted online from December 21, 2016, to December 29, 2016, and was sent twice via e-mail.

2.3. Statistical Analysis. All statistical analyses and data handling were performed using R version 3.4 (R Foundation for Statistical Computing, Vienna, Austria). The Chi-square 
test of independence was used to identify the statistical significance of characteristic differences between KMDs and KMNPSs groups. The characteristics include diagnostic methods, psychotropic drug use rates, and treatment methods, and treatment-related factors.

We divided the data into two groups, KMD and KMNPS, for comparative analysis, because it was assumed that there would be differences in treatment patterns between the two groups. Therefore, we tried to uncover the differences and use them as data to provide a complementary treatment process in the future.

\section{Results}

3.1. Demographic Characteristics. Statistical analysis was performed based on the survey data of 677 KMDs and 80 KMNPSs. The response rate was 3.69\% for KMDs and $48.5 \%$ for KMNPSs. The average age of the KMD who participated in the questionnaire was $35.2 \%$ in their $40 \mathrm{~s}$ and $32.9 \%$ in their $30 \mathrm{~s}$. In the KMNPS, the average age was in their $30 \mathrm{~s}$ with $63.8 \%$, followed by those in their $40 \mathrm{~s}$ with $30.0 \%$. The gender of the KMD was $80.1 \%$ in male, $19.9 \%$ in female, and of the KMNPS was $68.8 \%$ in male and $31.3 \%$ in female. The types of medical institutions worked were KM clinics $66 \%$, KM hospitals $17.1 \%$ and long-term care hospitals $6.9 \%$ in KMDs, and KM clinics 45\%, KM hospitals 28.8\%, and longterm care hospitals $6.9 \%$ in KMNPSs, respectively. It is shown that clinical career did not differ by $12.97 \pm 6.6$ years for the KMD and average of $12.18 \pm 8.64$ years for the KMNPS. The degrees in KMD were $54.4 \%$ for bachelor, $26.4 \%$ for doctor, and $19.2 \%$ for masters. The degree in KMNPS was $51.3 \%$ for doctor, $28.8 \%$ for master, and $20.0 \%$ for bachelor. Demographic characteristics of respondents are shown in Table 1.

The average number of patients with anxiety disorder per month was $4.47 \pm 4.56$ in the KMD and $6.63 \pm 5.72$ in the KMNPS. The average number of first-visit patients with anxiety disorder was $8.95 \pm 15.63$ in the KMD and $14.31 \pm 14.86$ in the KMNPS. It is shown in Table 2.

3.2. Diagnostic Methods of Anxiety Disorder Patients. In the $\mathrm{KMD}$, the diagnostic methods of patients with anxiety disorder were as follows: self-evaluation (87.7\%) and counseling (72.8\%), and they were as follows in the KMNPS: self-evaluation $(86.3 \%)$, counseling $(75.0 \%)$, DSM, ICD diagnostic criteria (56.3\%), anxiety questionnaire (e.g., STAI, BAI, and HAM-A) (55.0\%), instrumental examination (e.g., HRV, Neurofeedback, 33.3\%). Anxiety questionnaire, semi- or structured interview, DSM.KCD diagnostic criteria, and instrumental examination (e.g., HRV and Neurofeedback) were used more significantly $(p<0.001)$ by KMNPS (Table 3$)$.

3.3. Psychotropic Drug Use Rate in Anxiety Disorder Patients. The proportion of psychotropic medications in first-visit patients with anxiety disorder is almost the same as $48.31 \pm 32.27 \%$ of the KMD and $47.88 \pm 26.66 \%$ of the KMNPS (Table 4).
3.4. Treatments of Anxiety Disorder Patients. The reason why patients with anxiety disorder wants to be treated with oriental medicine is to stop or reduce the treatment of drugs (56.0\% and $80.0 \%$ ), improvement of physical symptoms (47.6\% and $78.8 \%$ ), improvement of psychological symptoms $(46.4 \%$ and $61.3 \%)$, and the quality of life $(46.4 \%$ and $43.8 \%$ ) in both the KMD and the KMNPS. KMNPS answered significantly $(p<0.001)$ more in the improvement of physical symptoms and stop medication or decrease dosage (Table 5).

The most common treatment for anxiety disorder in both KMD and KMNPS is acupuncture (KMD 95.9\% and KMNPS 98.8\%) and herbal decoction (KMD 85.1\% and KMNPS 91.3\%). In the KMD, treatments are used in the order of dry cupping (71.5\%), moxibustion (48.6\%), pharmaco-acupuncture (24.8\%). In the KMNPS, treatments are used in the order of psychotherapy (71.3\%), moxibustion (60.0\%), and NHIS exclusion herbal extracts (45.0\%). Electroacupuncture, NHIS-excluded herbal extracts, wet cupping, aromatherapy, and physical therapy were significantly $(p<0.001)$ more used in KMNPS (Table 6).

KMD mainly used self-evaluation (93.1\%) and counseling $(75.9 \%)$ to evaluate anxiety disorder treatment. KMNPS used self-evaluation (96.2\%), counseling (75.0\%), tapering of antipsychotics (58.8\%), and change in test scale $(52.5 \%)$. KMNPS tended to use $(p<0.001)$ more change in test scale, tapering of psychotropic medicine to assess treatment effects (Table 7).

The most important factor in the treatment of anxiety disorder in KMD was herbal medicine treatment (43.1\%) and rapport formation (39.9\%), while in KMNPS, herbal medicine treatment $(45.0 \%)$, patient's temperament $(21.3 \%)$, and rapport formation (20.0\%). Significant differences $(p<0.001)$ were found in the rapport formation with high response to KMD and patient's temperament with high response to KMNPS (Table 8 ).

The factors that interfere with the treatment of patients with anxiety disorders are patient symptom characteristics (49.9\%), patient lifestyle (44.6\%), patient family problem (43.9\%), and patient personality characteristics (39.6\%) in KMD. In the case of KMNPS, the factors are patient family problem $(68.8 \%)$, patient personality characteristics $(52.5 \%)$, patient symptom characteristics $(46.2 \%)$, and patient lifestyle and slow treatment effect $(37.5 \%)$. There was a significant difference $(p<0.001)$ in the patient family problems, which was highly answered by KMNPS (Table 9).

KMD chose the use of diagnostic tools the most (79.6\%) as an area requiring additional education in the treatment of anxiety disorder and was significantly higher than KMNPS $(p<0.001)$. On the other hand, KMNPS answered psychotherapy (62.5\%) as the most important additional education. Results are shown in Table 10.

\section{Discussion}

Before discussing the survey results, the KMD and KMNPS survey response rates were very different, $3.69 \%$ and $48.5 \%$, respectively. Since the subject of the survey is anxiety disorders, one of the most frequent diseases in the psychiatric 
TABLE 1: Demographic characteristics of respondents.

\begin{tabular}{lcc}
\hline Classification & KMD $(N=677)$ & $\begin{array}{c}\text { KMNPS }(N=80) \\
n(\%) / \text { mean } \pm \text { SD }\end{array}$ \\
\hline Age & & $0(0.0)$ \\
$\quad$ Less than 30 & $111(16.4)$ & $51(63.8)$ \\
$30-39$ & $223(32.9)$ & $24(30.0)$ \\
$40-49$ & $238(35.2)$ & $5(6.3)$ \\
$50-59$ & $5(0.7)$ & $0(0.0)$ \\
More than 60 & $18(2.7)$ & $25(31.3)$ \\
Sex & & $55(68.8)$ \\
Female & $135(19.9)$ & $36(45.0)$ \\
$\quad$ Male & $542(80.1)$ & $23(28.8)$ \\
Place of work & & $5(6.3)$ \\
$\quad$ Korean medical clinic & $449(66.3)$ & $16(2.0)$ \\
Korean medical hospital & $116(17.1)$ & $12.97 \pm 6.6$ \\
Convalescence hospital & $47(6.9)$ & \\
$\quad$ Others & $65(9.6)$ & $16(20)$ \\
Clinical experience & & $23(28.8)$ \\
$\quad$ Years & $12.18 \pm 8.64$ & $41(51.3)$ \\
Education & $368(54.4)$ & $130(19.2)$ \\
$\quad$ Bachelor & $179(26.4)$ & \\
Master & & \\
$\quad$ Doctor & & \\
\hline
\end{tabular}

Values are expressed as $n$ (\%) for age, sex, place of work, and education and as mean ( ${ }^{*}$ standard deviation) for clinical experience. KMD, Korean medicine doctor; KMNPS, Korean medicine neuropsychiatry specialist.

TABLE 2: Number of patients with anxiety disorders.

\begin{tabular}{lcc}
\hline Classification & KMD $(N=677)$ & KMNPS $(N=80)$ \\
$n(\%)$ & $n(\%)$ & \\
Number of patients diagnosed with anxiety disorder per month & $178(26.3)$ & $12(15.0)$ \\
0 & $461(68.1)$ & $52(65.0)$ \\
$1-9$ & $29(4.3)$ & $13(16.3)$ \\
$10-19$ & $5(0.7)$ & $3(3.8)$ \\
$20-29$ & $4(0.6)$ & $0(0.0)$ \\
More than 30 & $4.47(4.56)$ & $6.63(5.72)$ \\
Mean (SD) & & $64(12.4)$ \\
Number of patients complain of anxiety disorder as a symptom per month & $471(69.6)$ \\
0 & $97(14.3)$ & $39(48.8)$ \\
$1-10$ & $14(2.1)$ & $26(32.5)$ \\
$11-29$ & $6(0.9)$ & $7(8.8)$ \\
$30-49$ & $5(0.7)$ & $2(2.5)$ \\
$50-100$ & $8.95(15.63)$ & $0(0.0)$ \\
More than 100 & $14.31(14.86)$ \\
\hline
\end{tabular}

Values are expressed as $n$ (\%) and mean (standard deviation). KMD, Korean medicine doctor; KMNPS, Korean medicine neuropsychiatry specialist.

TABle 3: Diagnostic methods for patients with anxiety disorders.

\begin{tabular}{|c|c|c|c|}
\hline Method & $\begin{array}{c}\text { KMD }(N=677) \\
n(\%)\end{array}$ & $\begin{array}{c}\text { KMNPS }(N=80) \\
n(\%)\end{array}$ & $p$ value ( $\chi^{2}$ test $)$ \\
\hline Self-evaluation & $594(87.7)$ & $69(86.3)$ & 0.839 \\
\hline Anxiety questionnaire (e.g., STAI, BAI, HAM-A) & $78(11.5)$ & $44(55.0)$ & $<0.001$ \\
\hline Semi- or structured interview (e.g., SCID) & $16(2.4)$ & $10(12.5)$ & $<0.001$ \\
\hline DSM, KCD diagnostic criteria & $92(13.6)$ & $45(56.3)$ & $<0.001$ \\
\hline Counseling & $493(72.8)$ & $60(75.0)$ & 0.778 \\
\hline Instrumental examination (e.g., HRV and Neurofeedback) & $58(8.6)$ & $27(33.8)$ & $<0.001$ \\
\hline Etc. & $10(1.5)$ & $2(2.5)$ & 0.829 \\
\hline
\end{tabular}

Values are expressed as $n$ (\%). $p$ values are calculated by Chi-square test. KMD, Korean medicine doctor; KMNPS, Korean medicine neuropsychiatry specialist; STAI, state-trait anxiety inventory; BAI, Beck Anxiety Inventory; HAM-A, Hamilton Anxiety Rating Scale; SCID, structured clinical interview for DSM; KCD, Korean standard classification of diseases; HRV, heart rate variability. 
TABLE 4: Percentage of patients taking psychiatric medications.

\begin{tabular}{lcc}
\hline Classification & KMD $(N=677)$ & KMNPS $(N=80)$ \\
$n(\%)$ & $3(3.8)$ \\
\hline 0 & $n(\%)$ & $8(10.0)$ \\
$1-10 \%$ & $102(15.1)$ & $9(11.3)$ \\
$10-30 \%$ & $91(13.4)$ & $33(41.3)$ \\
$30-60 \%$ & $168(24.8)$ & $22(27.5)$ \\
$60-90 \%$ & $175(25.8)$ & $5(6.3)$ \\
Over than 90\% & $102(15.1)$ & $47.88(26.66)$ \\
Mean (SD) & $48.31(32.27)$ & \\
\hline
\end{tabular}

Values are expressed as $n$ (\%). KMD, Korean medicine doctor; KMNPS, Korean medicine neuropsychiatry specialist.

TABLE 5: The reason why patients want KM treatment.

\begin{tabular}{lccc}
\hline Reason & KMD $(N=677)$ & KMNPS $(N=80)$ & $n(\%)$ \\
& $n(\%)$ & $35(43.8)$ & $p$ value $\left(\chi^{2}\right.$ test $)$ \\
\hline Improving the quality of life & $314(46.4)$ & $49(61.3)$ & 0.743 \\
Improvement of psychological symptoms & $314(46.4)$ & $63(78.8)$ & 0.016 \\
Improvement of physical symptoms & $322(47.6)$ & $64(80.0)$ & $<0.001$ \\
Stop medication or decrease dosage & $379(56.0)$ & $39(48.8)$ & $<0.001$ \\
Dissatisfaction with modern medical treatment & $292(43.1)$ & $1(1.3)$ & 0.402 \\
Etc. & $7(1.0)$ & 1 \\
\hline
\end{tabular}

Values are expressed as $n$ (\%). $p$ values are calculated by chi-square test. KMD, Korean medicine doctor; KMNPS, Korean medicine neuropsychiatry specialist.

TABLE 6: KM treatment methods used for anxiety disorders.

\begin{tabular}{lccc}
\hline Treatment & KMD $(N=677)$ & KMNPS $(N=80)$ & $n(\%)$ \\
& $n(\%)$ & $79(98.8)$ & $p$ value $\left(\chi^{2}\right.$ test $)$ \\
\hline Acupuncture & $649(95.9)$ & $32(40.0)$ & 0.335 \\
Electroacupuncture & $132(19.5)$ & $12(15.0)$ & $<0.001$ \\
Ear acupuncture & $79(11.7)$ & $8(10.0)$ & 0.494 \\
Intradermal acupuncture & $42(6.2)$ & $2(2.5)$ & 0.292 \\
Warm acupuncture & $31(4.6)$ & $26(32.5)$ & 0.568 \\
Pharmacoacupuncture & $168(24.8)$ & $0(0.0)$ & 0.176 \\
Bee venom acupuncture & $21(3.1)$ & $14(17.5)$ & 0.216 \\
Chuna manipulation & $79(11.7)$ & $7(8.8)$ & 0.186 \\
Conduction exercise & $16(2.4)$ & $34(42.5)$ & 0.005 \\
56 NHIS inclusion herbal extracts & $178(26.3)$ & $36(45.0)$ & 0.003 \\
NHIS exclusion herbal extracts & $170(25.1)$ & $73(91.3)$ & $<0.001$ \\
Chinese herbal decoctions & $576(85.1)$ & $33(41.3)$ & 0.186 \\
Wet cupping & $147(21.7)$ & $28(35.0)$ & $<0.001$ \\
Dry cupping & $484(71.5)$ & $48(60.0)$ & 0.017 \\
Moxibustion & $329(48.6)$ & $27(33.8)$ & 0.07 \\
Aromatherapy & $66(9.7)$ & $5(6.3)$ & $<0.001$ \\
Biofeedback & $13(1.9)$ & $57(71.3)$ & 0.044 \\
Psychotherapy & $117(17.3)$ & $<0.001$ \\
\hline
\end{tabular}

Values are expressed as $n$ (\%). $p$ values are calculated by Chi-square test. KMD, Korean medicine doctor; KMNPS, Korean medicine neuropsychiatry specialist; NHIS, National Health Insurance Service.

field, KMNPS majoring in psychiatry seems to have higher interest and response rates of the questionnaire than KMD.

In the demographic context, KMNPS accounted for the highest percentage in the $30 \mathrm{~s}$ because the KMNPS began to produce in 2000 and have had a shorter history. In the case of KMNPS, there were a relatively large number of workers working at the KM Hospital which has specialized departments. The relatively large number of doctor and master degree holder in the specialist are seen as an effort to have medical expertise (Table 1).

The assessment is as follows. As a diagnostic/evaluation tool used with anxiety disorders, both KMD and KMNPS account for a high proportion of self-evaluation of patients and information acquisition through counseling. Unlike KMD, KMNPS is more likely to use objective indicators such as DSM/ICD diagnostic criteria, questionnaires, and 
TABLE 7: Assessment of treatment effect of anxiety disorder patients.

\begin{tabular}{lccc}
\hline Classification & KMD $(N=677)$ & KMNPS $(N=80)$ & $n(\%)$ \\
& $n(\%)$ & $77(96.2)$ & $p$ value $\left(\chi^{2}\right.$ test $)$ \\
\hline Self-evaluation & $63(93.1)$ & $42(52.5)$ & 0.396 \\
Change in test scale & $96(14.2)$ & $60(75.0)$ & $<0.001$ \\
Counseling & $514(75.9)$ & $47(58.8)$ & 0.965 \\
Tapering of psychotropic medicine & $260(38.4)$ & $13(16.2)$ & $<0.001$ \\
Instrumental examination & $62(9.2)$ & $22(27.5)$ & 0.07 \\
Pulse diagnosis & $156(23.0)$ & $0(0)$ & 0.453 \\
Etc. & $7(1.0)$ & 1 \\
\hline
\end{tabular}

Values are expressed as $n$ (\%). $p$ values are calculated by Chi-square test. KMD, Korean medicine doctor; KMNPS, Korean medicine neuropsychiatry specialist; NHIS, National Health Insurance Service.

TABLE 8: The most important factor in treating anxiety disorders.

\begin{tabular}{lccc}
\hline Factor & KMD $(N=677)$ & KMNPS $(N=80)$ & $n(\%)$ \\
Rapport formation & $n(\%)$ & $16(20.0)$ & $p$ value $\left(\chi^{2}\right.$ test $)$ \\
Herbal medicine treatment & $270(39.9)$ & $36(45.0)$ & $<0.001$ \\
Acupuncture treatment & $292(43.1)$ & $5(6.3)$ & 0.842 \\
Patient's temperament & $52(7.7)$ & $17(21.3)$ & 0.815 \\
Psychotherapy & $37(5.5)$ & $6(7.5)$ & $<0.001$ \\
Psychotropic medication & $14(2.1)$ & $0(0)$ & 0.013 \\
\hline
\end{tabular}

Values are expressed as $n$ (\%). $p$ values are calculated by Chi-square test. KMD, Korean medicine doctor; KMNPS, Korean medicine neuropsychiatry specialist.

TABLE 9: Obstructive factors in the treatment of anxiety disorders.

\begin{tabular}{lccc}
\hline Factor & KMD $(N=677)$ & KMNPS $(N=80)$ & $p(\%)$ \\
& $n(\%)$ & $37(46.2)$ & $p$ value $\left(\chi^{2}\right.$ test $)$ \\
\hline Patient symptom characteristics & $338(49.9)$ & $42(52.5)$ & 0.614 \\
Patient personality characteristics & $268(39.6)$ & $30(37.5)$ & 0.036 \\
Patient lifestyle & $302(44.6)$ & $55(68.8)$ & 0.275 \\
Patient family problems & $207(43.9)$ & $23(28.7)$ & $<0.001$ \\
Alcohol dependence & $161(23.8)$ & $21(26.2)$ & 0.4 \\
Accompanying physical disease & $101(14.9)$ & $30(37.5)$ & 0.014 \\
Slow treatment effect & $177(26.1)$ & $21(26.2)$ & 0.043 \\
Distrust of treatment & $157(23.2)$ & $27(33.8)$ & 0.638 \\
Inadequate expectations & $175(25.8)$ & $26(32.5)$ & 0.168 \\
Lack of motivation & $181(26.7)$ & $23(28.7)$ & 0.336 \\
Lack of rapport formation & $161(23.8)$ & $2(2.5)$ & 0.4 \\
Others & $18(2.7)$ & 1 \\
\hline
\end{tabular}

Values are expressed as $n$ (\%). $p$ values are calculated by chi-square test. KMD, Korean medicine doctor; KMNPS, Korean medicine neuropsychiatry specialist.

TABle 10: Additional training items needed for anxiety disorders treatment.

\begin{tabular}{lccc}
\hline Training item & KMD $(N=677)$ & KMNPS $(N=80)$ & $n(\%)$ \\
\hline Use of diagnostic tool & $n(\%)$ & $34(42.5)$ & $p$ value $\left(\chi^{2}\right.$ test $)$ \\
Use of instrumental examination & $539(79.6)$ & $20(25.0)$ & $<0.001$ \\
Use of psychotherapy & $222(32.8)$ & $50(62.5)$ & 0.198 \\
Details of psychiatry & $356(49.6)$ & $37(46.2)$ & 0.039 \\
Etc. & $253(37.4)$ & $6(7.5)$ & 0.155 \\
\hline
\end{tabular}

Values are expressed as $n$ (\%). $p$ values are calculated by Chi-square test. KMD, Korean medicine doctor; KMNPS, Korean medicine neuropsychiatry specialist. 
physical tests (Table 3 ). This can be seen as securing more patient information and providing objectivity of secured information.

The rate of psychotropic medication in patients with anxiety disorder was $48 \%$ in both groups, and the reason seems that the severity of the symptoms of anxiety disorder patients coming to KMD and KMNPS for treatment is considered to be the same (Table 4).

The reason for choosing KM treatment for anxiety disorders is the improvement of psychological and physical symptoms, along with cutting off or reducing the amount of psychotropic drugs. Although psychotropic medication is a universal treatment for the treatment of anxiety disorders, patients are often found to seek Korean medicine for reducing their reliance on psychotropic drugs or as an alternative treatment for adverse drug reactions (Table 5).

For the treatment tools used in the treatment of anxiety disorders, both KMD and KMNPS selected the treatment tools commonly used in Korean medicine such as acupuncture and herbal medicine. However, KMNPS relatively chose psychotherapy which requires professionalism in terms of its application (Table 6).

The most commonly used methods for evaluating the effectiveness of treatments are self-evaluation of patients and counseling in both KMD and KMNPS. In addition, KMNPS are more likely to use objective indicators such as psychotropic drug doses and changes in psychological scale measures; it is consistent with the characteristics that use objective indicators (Tables 3 and 7).

For the important factors in the treatment of anxiety disorders, both groups regarded herbal medicine treatment as the most important. While KMD emphasized herbal medicine treatment and rapport formation at an equal level, in the case of KMNPS, the treatment of herbal medicine is the most important, followed by the patient's temperament and the rapport formation (Table 8). KMNPS tend to prefer psychotherapy over KMD in the treatment of anxiety disorders (Table 6) and seem to focus on resources and making cooperative relationship with patients.

In questionnaire of the disturbances in the treatment of anxiety disorders, both groups reported that the various factors presented are meaningful. In KMNPS, the family problems, personality characteristics, accompanying physical illnesses, and slow treatment effects are considered as obstacles. In particular, the family problems and personality characteristics of patients are $68.8 \%$ and $52.5 \%$, respectively, that is over half of them (Table 9). While current symptoms are the most important factor for KMD, KMNPS tends to place greater importance on family problems and personality traits in patients. Just as KMNPS consider the patient's temperament as an important factor in treatment, they can be thought to focus on factors that are more problematic than the symptoms themselves.

Finally, in the additional educational items that they think are necessary, KMD choose utilizing diagnostic tools the most and KMNPS choose psychotherapy the most (Table 10). This may mean that KMD is not accustomed to the Western neuropsychiatric diagnosis as compared to KMNPS. In the case of KMNPS, since they mainly used psychotherapy to treat patients with anxiety disorders (Table 6), so the need for new psychotherapeutic education appears to be for additional expertise.

There are several limitations in this survey. We surveyed the current state of KM treatment for anxiety disorders only in Korea. Therefore, China, Japan, and many other countries using traditional Asian medicine (TAM) have not been able to figure out how they perceive and treat anxiety disorders. In addition, this was an overview of how KMDs diagnose, treat, and evaluate anxiety disorders. It does not include specific and practical contents such as which acupuncture points, prescriptions, or psychotherapies are used frequently. Therefore, various treatment methods and patterns that are used empirically in the clinical field should be additionally considered in future survey for development of CPG.

This study is the first study on the current state of the medical practice of the TAM psychiatrist in Korea. The guideline currently under development will include the characteristics such as the rate of anxiety disorders patients in $\mathrm{KM}$ institution, their psychotropic drug use, and $\mathrm{KM}$ treatment tool type and utilization tendency. And this study illustrated the differences between KMD and KMNPS for anxiety disorders, so that it was possible to check what is needed to improve the ability for treating anxiety disorders. In the future, based on the results of this study, statistical studies of other diseases are also expected to be more diverse and in-depth analysis than before through comparison of specialists and general practitioners.

\section{Conclusions}

This study is the first study to analyze the current state of anxiety disorder treatment in KMDs. KMD and KMNPS showed similar patterns in the perception, diagnosis, and treatment of anxiety disorders, as both are studied and trained in the same oriental medicine. However, the KMNPS is more likely to use objective indicators and psychotherapies as they have been trained in neuropsychiatry for three years and has had experience in applying expertise to the treatment of anxiety disorders. Further clinical research is required to develop clinical guidelines utilized by KMD and KMNPS, respectively.

\section{Abbreviations}

BAI: Beck Anxiety Inventory

CPG: $\quad$ Clinical practice guideline

DSM: Diagnostic and statistical manual of mental disorders

GAD: $\quad$ Generalized anxiety disorder

HAM-A: Hamilton Anxiety Rating Scale

HRV: Heart rate variability

ICD: International Classification of Diseases

KM: $\quad$ Korean medicine

KMD: Korean medicine doctor

KMNPS: Korean medicine neuropsychiatric specialist

NHIS: National Health Insurance Service 
PD: $\quad$ Panic disorder

PTSD: Posttraumatic stress disorder

SAD: $\quad$ Social anxiety disorder

SCID: $\quad$ Structured Clinical Interview for DSM

STAI: State-trait anxiety inventory

TAM: Traditional Asian Medicine.

\section{Data Availability}

The data used to support the findings of this study are available from the corresponding author upon request.

\section{Conflicts of Interest}

The authors declare that there are no conflicts of interest exist.

\section{Authors' Contributions}

SIS and GWK conceived the study. SIS retrieved relevant literatures and collected questionnaires. SIS, SYC, EJK, and GWK constructed the questionnaire. SIS, SYC, EJK, BSK, HWJ, and GWK revised the questionnaire and drafted the manuscript. GHL organized and supervised the data analysis and provided critical comments on various drafts of the paper. GWK supervised the study. All authors read and approved the final manuscript.

\section{Acknowledgments}

This study was supported by the Korea Health Industry Development Institute (HB16C0021).

\section{Supplementary Materials}

Survey for the Development of Standard Clinical Pathway Guideline for Korean Medicine with Anxiety Disorders. (Supplementary Materials)

\section{References}

[1] World Health Organization, Depression and Other Common Mental Disorders: Global Health Estimates, World Health Organization, Geneva, Switzerland, 2017.

[2] GBD 2015 Disease and Injury Incidence and Prevalence Collaborators, and others, "Global, regional, and national incidence, prevalence, and years lived with disability for 310 diseases and injuries, 1990-2015: a systematic analysis for the global burden of disease study 2015," The Lancet, vol. 388, no. 10053, pp. 1545-1602, 2016.

[3] Z. Steel, C. Marnane, C. Iranpour et al., "The global prevalence of common mental disorders: a systematic review and metaanalysis 1980-2013," International Journal of Epidemiology, vol. 43, no. 2, pp. 476-493, 2014.

[4] Ministry of Health and Welfare, The Epidemiological Survey of Mental Disorders in Korea, Ministry of Health and Welfare, New Delhi, India, 2016.

[5] National Center for Mental Health, National Mental Health Statistsics 2018, National Center for Mental Health, Mandaluyong, Philippines, 2018.
[6] S. K. Eom, M. S. Shin, and S. J. Kwon, "A study on the legislations and amendments of the medical and pharmaceutical laws and regulations-focusing on the duties of Korean (oriental) medicine doctors and Korean (oriental) pharmacists as well as the public health system," The Journal of Korean Medical History, vol. 26, no. 2, pp. 175-185, 2013.

[7] J. D. Kim and M. S. Son, National Health Insurance Statistical Yearbook, Health Insurance Review and Assessment Service and National Health Insurance Service, Seoul, Korea, 2014.

[8] Ministry of Health and Welfare (Korea), Research Report on Post-implementation Evaluation and Activation Plan of Korean Medicine Specialist Board System, Ministry of Health and Welfare, Sejong, Korea, 2014.

[9] H. S. Lee and M. S. Koo, "Modern psychiatric understanding of the psychopathology of psychosis in oriental medicine," Journal of Korean Neuropsychiatric Association, vol. 49, pp. 508-515, 2010.

[10] World Health Organization, WHO Global Report on Traditional and Complementary Medicine 2019, World Health Organization, Geneva, Switzerland, 2019.

[11] China Traditional China Medicine Publisher, Evidence-based Guidelines of Clinical Practice in Chinese Medicine: Internal Medicine, China Traditional China Medicine Publisher, Beijing, China, 2011.

[12] C. Alexander and J. Fraser, "General practitioners' management of patients with mental health conditions: the views of general practitioners working in rural north-western New South Wales," Australian Journal of Rural Health, vol. 16, no. 6, pp. 363-369, 2008.

[13] A. Ehlers, N. Gene-Cos, and S. Perrin, "Low recognition of post-traumatic stress disorder in primary care," London Journal of Primary Care, vol. 2, no. 1, pp. 36-42, 2009.

[14] L. J. Hipol and B. J. Deacon, "Dissemination of evidencebased practices for anxiety disorders in Wyoming," Behavior Modification, vol. 37, no. 2, pp. 170-188, 2013.

[15] A. F. Jorm, A. J. Morgan, and A. Wright, "Interventions that are helpful for depression and anxiety in young people: a comparison of clinicians' beliefs with those of youth and their parents," Journal of Affective Disorders, vol. 111, no. 2-3, pp. 227-334, 2008.

[16] A. F. Jorm, A. J. Morgan, and A. Wright, "Actions that young people can take to prevent depression, anxiety and psychosis: beliefs of health professionals and young people," Journal of Affective Disorders, vol. 126, no. 1-2, pp. 278-281, 2010.

[17] M. Kartal, O. Coskun, and N. Dilbaz, "Recognizing and managing anxiety disorders in primary health care in Turkey," BMC Family Practice, vol. 11, no. 30, 2010.

[18] A. A. McAleavey, L. G. Castonguay, and M. R. Goldfried, "Clinical experiences in conducting cognitive-behavioral therapy for social phobia," Behavior Therapy, vol. 45, no. 1, pp. 21-35, 2014.

[19] A. J. Morgan, A. F. Jorm, and N. J. Reavley, "Beliefs of Australian health professionals about the helpfulness of interventions for mental disorders: differences between professions and change over time," Austrian \& New Zealand Journal of Psychiatry, vol. 47, no. 9, pp. 840-848, 2014.

[20] J. B. Nissen and P. H. Thomsen, "Clinicians' views on clinical examination and treatment of children and adolescents with obsessive-compulsive disorder (OCD). A Danish national survey study," Nordic Journal of Psychiatry, vol. 62, no. 4, pp. 309-314, 2008.

[21] D. Sars and A. van Minnen, "On the use of exposure therapy in the treatment of anxiety disorders: a survey among 
cognitive behavioural therapists in The Netherlands," $B M C$ Psychology, vol. 3, no. 26, 2015.

[22] L. E. Szkodny, M. G. Newman, and M. R. Goldfried, "Clinical experiences in conducting empirically supported treatments for generalized anxiety disorder," Behavior Therapy, vol. 45, no. 1, pp. 7-20, 2014.

[23] A. W. Wolf and M. R. Goldfried, "Clinical experiences in using cognitive-behavior therapy to treat panic disorder," Behavior Therapy, vol. 45, no. 1, pp. 36-46, 2014. 